\title{
Smartphone assist deep neural network model to recognize the high-quality tea using leaf maturity and its effect on leaf chlorophyll
}

\author{
Utpal Barman* \\ Girijananda Chowdhury Institute of Management and Technology, Guwahati (Assam), India \\ Ridip Dev Choudhury \\ Krishna Kanta Handiqui State Open University, Guwahati (Assam), India \\ Bipul Kumar Talukdar \\ Girijananda Chowdhury Institute of Management and Technology, Guwahati (Assam), India \\ George Bhokta \\ Girijananda Chowdhury Institute of Management and Technology, Guwahati (Assam), India \\ Sahrul Alom Choudhari \\ Girijananda Chowdhury Institute of Management and Technology, Guwahati (Assam), India \\ Abhinab Saikia \\ Girijananda Chowdhury Institute of Management and Technology, Guwahati (Assam), India \\ ${ }^{*}$ Corresponding author. Email: utpalbelsor@gmail.com
}

\section{Article Info}

https://doi.org/10.31018/

jans.v13i4.2950

Received: September 22, 2021

Revised: October 28, 2021

Accepted: November 4, 2021

\section{How to Cite}

Barman, U. et al. (2021). Smartphone assist deep neural network model to recognize the high-quality tea using leaf maturity and its effect on leaf chlorophyll. Journal of Applied and Natural Science, 13(4), 1249 - 1255. https://doi.org/10.31018/jans.v13i4.2950

\begin{abstract}
Immature and tender tea leaves always produce high-quality tea than mature tea leaves. Depending on the maturity and age of the leaf, the colour and texture of the tea leaf are different. The photosynthesis capacity of the tea leaf also changes with the change of leaf maturity. Though the tea farmer plucks, classifies, and recognizes the best tea leaves (immature and tender) by viewing the visual symptoms and position of the leaves, the method is not authentic all time and leads to the overall degradation of the tea quality. The present study presents a smartphone assist tea leaf recognition system by analyzing the colour and texture properties of the tea leaf. The six different colour features and 4 Haralick texture features were extracted in the colour and grey domain of the leaf images. Three types of tea leaves, i.e., mature, immature, and tender, were classified using Deep Neural Network (DNN) with ADAM (Adaptive Moment Estimation) optimizer. With an accuracy of $97 \%$, the DNN outperformed the Support Vector Machine (SVM) and K Nearest Neighbor (KNN). The SVM and KNN reported a total of $94.42 \%$ and $95.53 \%$ accuracy, respectively. The investigated system using DNN with an average precision and recall value of 98.67 and 98.34 , respectively, may detect and classify the tea leaf maturity status. The system also can be used in Al-based tea plucking robotic systems or machines.
\end{abstract}

Keywords: Agri-informatics, ANN, DNN, Leaf maturity, Precision Farming, Smartphone

\section{INTRODUCTION}

Assam is known for tea farming (Baruah, 2015). The immature and tender tea leaves are best for the tea industry and it produces more high-quality tea than mature tea leaves (Baruah, 2015). The tea farmer and gardener recognize the immature and tender tea leaves by viewing the visual symptom or by touching the leaf. Though the gardeners or workers are already well trained in this field, they may make mistakes during tea leaves plucking. Nowadays, artificial intelligence (AI) based agricultural robot is used by farmers in their farming. The application of modern technology such as machine learning in Agri-informatics has been broadly used for the last 5 years. Researchers have tried to use machine learning algorithms in the different areas of Agri-informatics such as soil texture analysis (Barman and Choudhury, 2019), soil pH prediction (Barman et al., 2008), chlorophyll prediction (Barman and Choudhury, 2020), and plant disease detection(Barman et al., 2020; Sood and Singh, 2020), etc.

The application of machine learning and deep learning algorithms for plant leaf maturity status recognition and classification is very limited. The researchers are found 
in fruit maturity status classification using machine learning techniques. Najeeb and Safar (2018) classified fruit maturity based on colour and texture. With an accuracy of $95.11 \%$ using deep learning, Mubin et al. (2019) classified young and mature oil palm trees. By extracting the colour and texture features. Behera et al. (2020) classified the maturity status of the papaya fruits using Artificial Neural Network (ANN). The use of $K$ Nearest Neighbor (KNN) was reported by Pourdarbani et al. (2020) for fuji apple and mango fruit classification by extracting the colour and texture features. Sudana et al. (2020) reported the application of the Convolutional Neural Network (CNN) for mangosteen fruit maturity status classification

Along with the change of colour and texture of the fruit, the above literature also focused on the extraction of colour and texture features of the fruits. Like fruit, the colour and texture features of the tea leaf also change with its maturity. The colour of the tender and immature leaves is lighter green than the mature leaves and these leaves have a smoothy texture than the mature leaves. Wicaksono et al. (2019) reported extracting $\mathrm{YCbCr}$ colour features to classify the tea leaf maturity. Like other farming, Artificial intelligence-enabled modern technology can be used in the tea plucking industry to increase the overall productivity of tea farming by reducing human error in the tea plucking process. Keep in mind the above issues, the present study presents a smart farming system to recognize the best tea leaf by classifying the maturity of tea leaves using image processing and Deep Neural Network (DNN).

\section{MATERIALS AND METHODS}

\section{Assam tea sample collection}

The tea images used in this study were collected from the tea gardens of lower Assam, especially the Kokrajhar tea estate. The tea farmers usually recognized and classified the maturity status of the leaves by using naked eyes and expertise. Anwar et al. (2017) reported the three classes of leaves of Aquilaria Beccariana based on the position of the leaves from the branch. Barman and Choudhury (2020) reported that

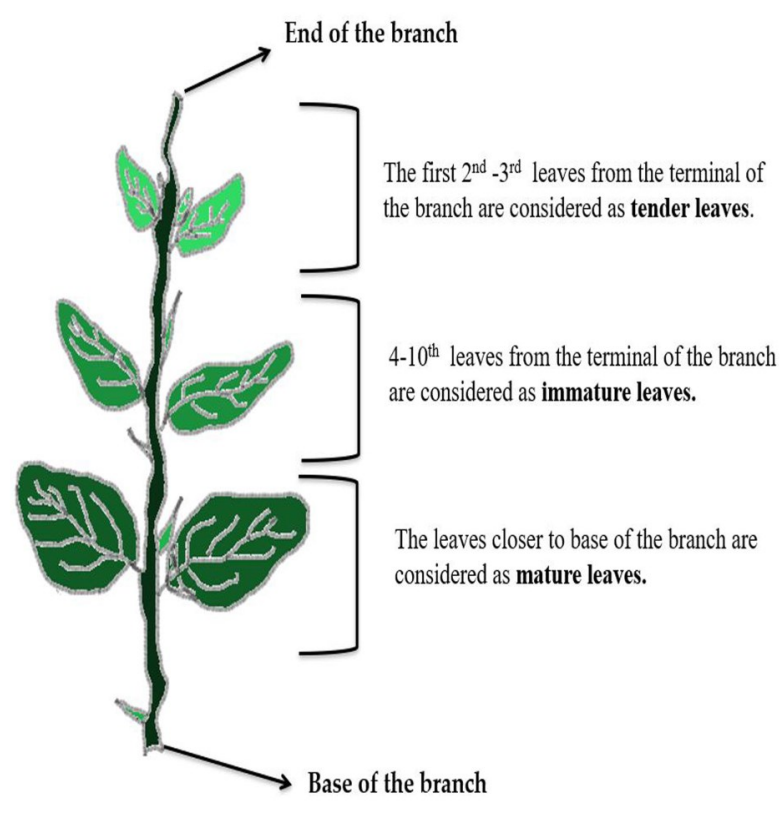

Fig. 1. Visual texture of the mature, immature, and tender tea leaf

the leaves closer to the branch's base are considered tea mature leaves, whereas $4-10$ th leaves from the terminal of the branch are usually considered immature tea leaves. The first $2-3^{\text {rd }}$ leaves from the terminal of the branch are considered tender tea leaves. In the present study, the tea leaves were plucked based on the study of Barman and Choudhury (2020) and reported three different categories of tea leaves by analyzing the visual texture of the leaves (Fig. 1).

After plucking, the images of the tea leaves were snapped using a smartphone by maintaining a 12-inch distance between the camera lens and tea leaf with the help of an easy measure distance-measuring app. In the dataset, out of 360 images, each of 120 tea images belonged to the mature, immature, and tender category of the tea leaves (Fig. 2). For the classification, the leaves were labelled as 0 for tender, 1 for immature, and 2 for mature (Fig. 2). For the training and testing of tea leaf images, an 80:20 (288:72 images) ratio was used. The overall process of the system was followed as Fig. 3.
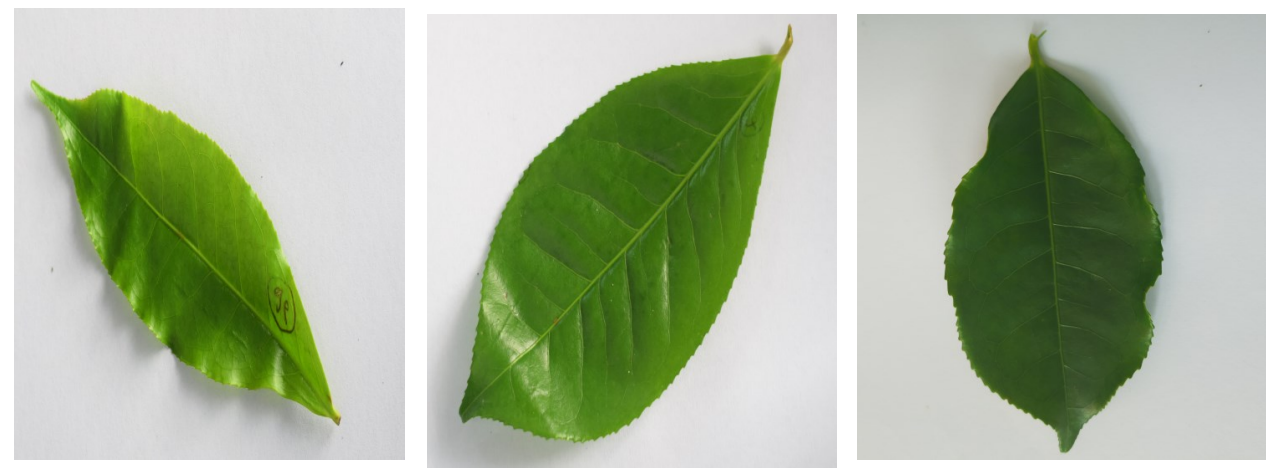

Fig. 2. a) Tender b) Immature c) Mature 


\section{Tea leaf maturity and its chlorophyll}

By analyzing the visual texture of the tea leaf, one can say that the greenness of the mature leaves was more than the immature and tender leaves, but the greenness of the immature and tender leaves was the same. The chlorophyll of each leaf was estimated using a Soil Plant Analysis Development (SPAD) meter (Barman, 2021; Barman et al., 2021; Barman and Choudhury, 2020). Most of the tender leaves' chlorophyll was in the range of 16 to 25 , whereas the immature leaves' chlorophyll values were in the range of 27 to 35 . The mature leaves' chlorophyll values were in the range of 40 to 60 . It was shown that the range of leaf chlorophyll was changing with the change of leaf maturity. The tender leaves have low chlorophyll compared to the mature leaves. It means that leaf maturity has a great effect on chlorophyll value. So, the age of the leaf changes the chlorophyll indexes of a leaf.

\section{Tea image pre-processing and feature extraction}

Farmers often check the maturity status of the tea leaves with naked eyes by analyzing the colour and texture of the leaves. During the dataset collection, it was found that the texture of the tea leaf varies from leaf to leaf. Before the texture extraction, images were converted to BGR to RGB format using CV2 of python. Then RGB images were again converted to GRAY scale to find the Haralick texture of the images. After the image conversion into colour and gray domain, the features were calculated by defining the 10 different features in the colour and gray domain. In the colour domain, the mean and standard deviation of red, green, and blue colours were determined. In the gray domain, the contrast, correlation, inverse difference moments (IDM), and entropy of the Haralick were calculated. The equations of the feature extraction method are presented below.

$$
\begin{aligned}
& \text { Mean of RGB: } \quad \sum_{i, j=0}^{N-1} i . p_{i, j} \quad \text {....Eq.1 } \\
& \text { Standard Deviation of RGB: } \left.\sum_{i, j=0}^{N-1}(i-\text { Mean })^{2} P(i, j)\right)^{1 / 2} \\
& \text { Contrast: } \quad \sum_{i, j=0}^{N-1}(i, j)^{2} P(i, j) \\
& \text { Correlation: } \sum_{i, j=0}^{N-1} P_{i j} \frac{((i-\mu)(j-\mu))}{\sigma^{2}} \quad \text {.....Eq.4 } \\
& \text { IDM: } \quad \sum_{i, j=0}^{N-1} \frac{1}{1+(i-j)^{2}} P(i, j) \\
& \text { Entropy: } \quad \sum_{i, j=0}^{N-1} P(i, j) \ln P(i, j)
\end{aligned}
$$

The algorithm of the feature extraction is given below. Algorithm: Haralick Feature of the tea Leaf image Step 1: Convert the colour image into RGB format. Step 2: Convert the RGB image into a gray format Step 3: Compute the Haralick Texture Feature Step 3.1: Compute colour feature from the RGB image i.e., the Mean of Red, Green, and Blue.

Step 3.2: Compute the $2^{\text {nd }}$ colour feature i.e., the Standard Deviation of Red, Green, and Blue.

Step 3.3: Compute the texture feature from the gray image i.e., contrast, correlation, inverse difference moments, and entropy.

Step 4: Compute the final feature vector by taking 6 colour features and 4 texture features from the Haralick definition.

From the single tea leaf, a total of 6 colour features were extracted in the colour domain(Barman, 2021) and the size of the feature vector of a single tea image was $1 \times 6$. The length of the Haralick texture feature for a single tea image was $1 \times 4$. The total feature vector size of a single tea image was $1 \times 10$. The mean values of the different features are shown in Table 1 and Fig. 4. In the present study, 288 tea leaf images were used for training purposes, so the size of the training feature vector of the maturity status classification was $288 \times 10$ with a testing size of $96 \times 10$.

\section{Tea maturity status classification using DNN}

In the present study, the DNN was used to classify the maturity of tea leaves. The DNN was introduced by considering 4 dense layers. The first dense layer received the 10 input feature parameters and it contains 512 hidden neurons. The second hidden layer and third

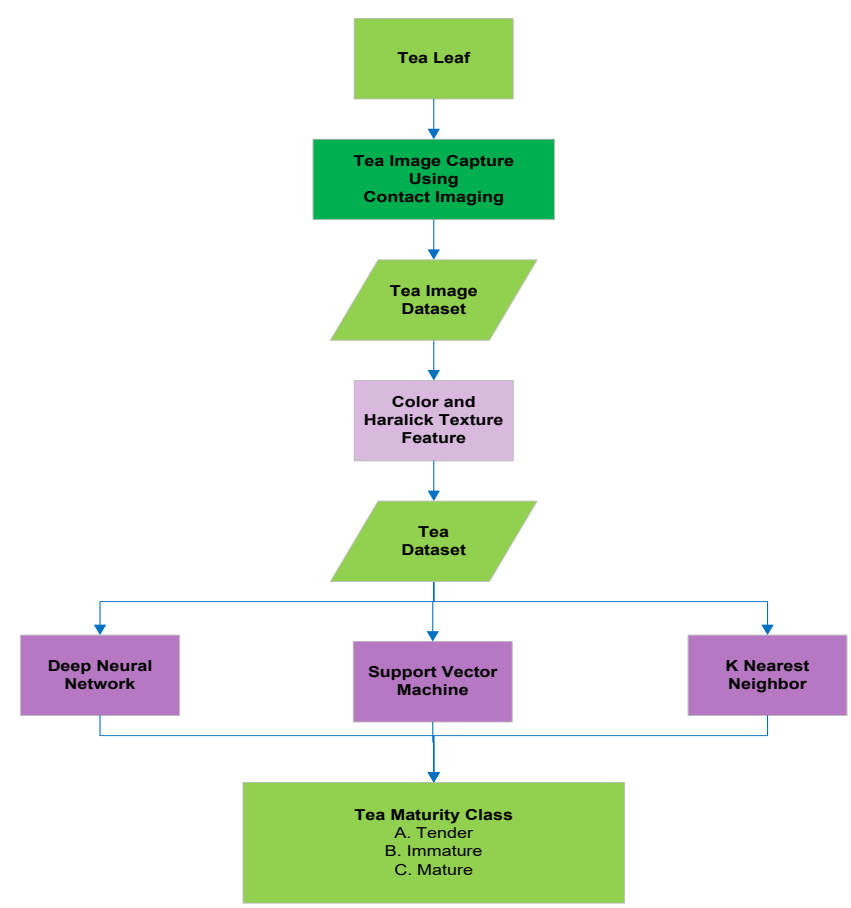

Fig. 3. Flow chart of the proposed system 


\begin{tabular}{llll}
\hline Table 1. Feature values of the tea leaf & & & \\
\hline Leaf types & Immature & Mature & Tender \\
\hline Mean_r & 108.0347 & 91.30356 & 93.80403 \\
Mean_g & 200.18 & 202.517 & 201.4312 \\
Mean_b & 12.01872 & 11.14845 & 11.57137 \\
Stddev_r & 8.49844 & 10.13689 & 8.314974 \\
Stddev_g & 8.936729 & 9.610005 & 9.191333 \\
Stddev_b & 2.420448 & 4.413765 & 2.896424 \\
Contrast & 5.616073 & 11.19486 & 7.653708 \\
Correlation & 0.942483 & 0.91271 & 0.918703 \\
IDM & 0.476161 & 0.382073 & 0.431297 \\
Entropy & 7.904473 & 8.579689 & 8.106591 \\
\hline
\end{tabular}

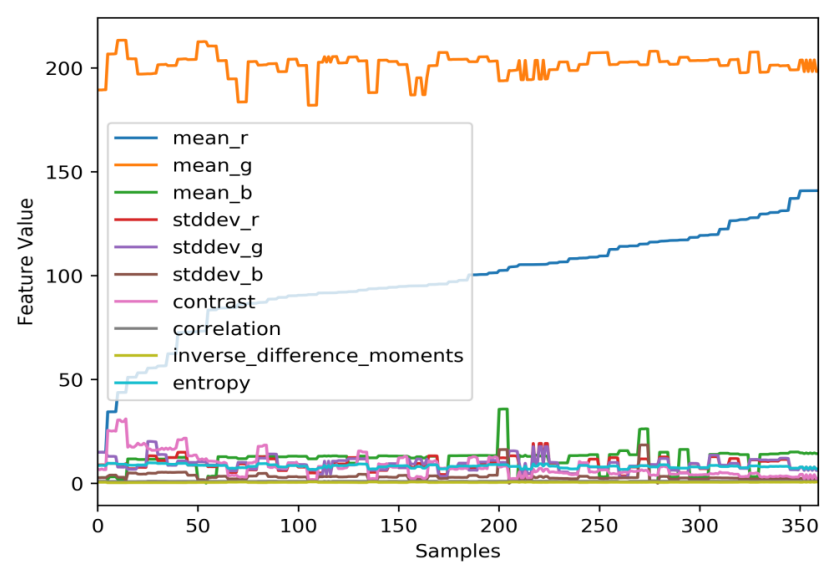

Fig. 4. Scatter plot of the different features of the tea leaf images

hidden layer contain the again 512 and 256 hidden neurons. The final dense layer contains 3 hidden layers for the classification of tea maturity. Except for the final dense layer, a relu activation function was added in the other layers to get the desired output. By applying two dropouts with a dropout rate of 0.2 and 0.3 , the model was tried to reduce the overfitting. With a learning rate of 0.001 , batch size $=10$, and epoch $=10$, the model was optimized with the ADAM (Adaptive Moment Estimation) optimizer. The loss of the DNN was calculated using the sparse categorical cross-entropy loss. For the better evaluation of the DNN model, another 20\% (29 samples) of the training feature (288 samples) were used for validation.

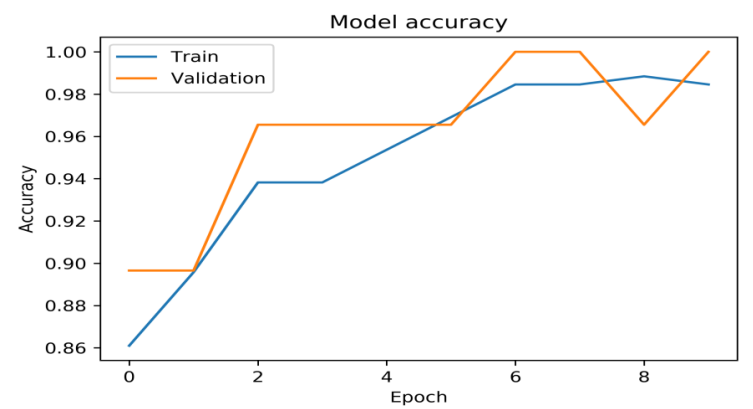

Tea leaf maturity classification using SVM and KNN The other state-of-the-art models, such as Support Vector Machine (SVM) and K Nearest Neighbor (KNN) were also applied to classify the tea leave's maturity. Two kernel parameters, such as liner and RBF were tunned with the SVM. The SVM-linear was applied by considering the $\mathrm{C}$ (Cost of misclassification) $=1$ whereas the results of the SVM-RBF were checked for the gamma values of $0.0001,0.001,0.01,0.1,1,10$, and 100.

The KNN classifier is a lazy classifier that follows the instance-based learning process. The $\mathrm{KNN}$ is a memory-based algorithm, so the classification was also done for the different $K$ values of 1 to 9 by considering the euclidian distance metrics and leaf size $=30$. Both the algorithms were implemented in the python environment using the Keras library.

\section{RESULTS AND DISCUSSION}

In the first phase, the classification of tea leaf maturity is done using a deep neural network. In the next step, the results are compared with other states of art algorithms.

Earlier in the above section, it is mentioned that the DNN model is optimized with the ADAM optimizer, and trained with a learning rate of 0.001 for 10 epochs. The overall validation of the model with the training set is presented below in Fig. 5. The performance of the DNN

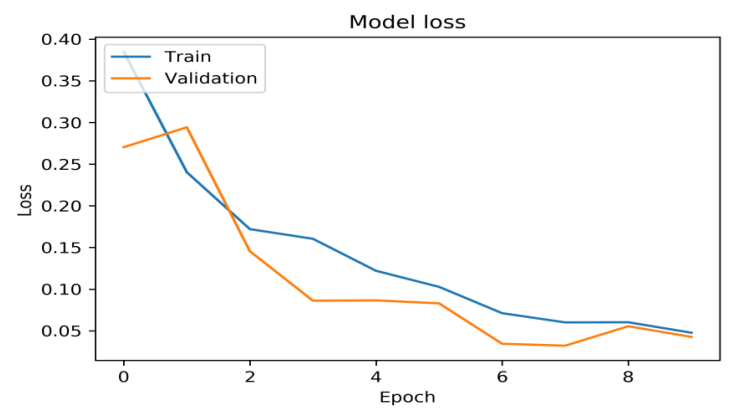

Fig. 5. Train and validation accuracy and error of the DNN model. 
Barman, U. et al. / J. Appl. \& Nat. Sci. 13(4), 1249-1255 (2021)

\begin{tabular}{|c|c|c|c|c|c|c|c|c|}
\hline \multirow[t]{2}{*}{ Model } & \multirow{2}{*}{$\begin{array}{l}\text { Class } \\
0\end{array}$} & \multirow{2}{*}{$\begin{array}{l}\text { Precision } \\
0.96\end{array}$} & \multirow{2}{*}{$\begin{array}{l}\text { Recall } \\
1\end{array}$} & \multirow{2}{*}{$\begin{array}{l}\text { F1 Score } \\
0.98\end{array}$} & \multirow[t]{2}{*}{ Accuracy } & \multicolumn{3}{|c|}{ Confusion Matrix } \\
\hline & & & & & & 26 & 0 & 0 \\
\hline \multirow[t]{2}{*}{ DNN } & 1 & 1 & 1 & 1 & 97 & 0 & 25 & 0 \\
\hline & 2 & 1 & 0.95 & 0.98 & & 1 & 0 & 20 \\
\hline
\end{tabular}

Table 3. Results of the SVM

\begin{tabular}{llll}
\hline Model & C Value & Gamma Value & Accuracy \\
\hline SVM-Linear & 1 & Auto & $42 \%$ \\
SVM-RBF & 1 & 0.0001 & $30.55 \%$ \\
SVM-RBF & 1 & 0.001 & $40.27 \%$ \\
SVM-RBF & 1 & 0.01 & $45.83 \%$ \\
SVM-RBF & 1 & 0.1 & $83.33 \%$ \\
SVM-RBF & 1 & 1 & $94.42 \%$ \\
SVM-RBF & 1 & 10 & $92.41 \%$ \\
SVM-RBF & 1 & 100 & $90.44 \%$ \\
\hline
\end{tabular}

model is also evaluated using the confusion matrix (Table 2). In Table 2, $100 \%$ perfect classification is reported in tender and immature tea leaves, whereas 1 image of mature tea leaf was misclassified as tender tea leaf. Along with the confusion matrix, the results of the DNN model are evaluated by calculating the precision, recall, and $\mathrm{f} 1$ score (Table 3 ). With a recall value of $0.98,1$, and 0.98 , the precision value of the model, for class 0,1 , and 2 is $0.96,1$, and 1 . The overall result of the model is $97 \%$.

The other state-of-the-art models, such as Support Vector Machine (SVM) and K Nearest Neighbor (KNN), are also applied in the model. By considering the $C=1$ and different gamma values, the SVM is tunned linear and RBF kernels and the results are presented in Table 3. Along with the decreasing performance of SVM Linear, the SVM RBF performance also decreases with the increasing value of gamma from 1 to 100 (Table 3). Table 3 indicates that the SVM model performs best $(94.42 \%)$ at $\mathrm{C}=1$ and gamma $=1$

The KNN algorithm is already reported by Behera et al. (2020) for papaya fruit maturity status classification with an accuracy of $100 \%$. Based on the study presented by Behera et al. (2020), the KNN algorithm was also applied to classify the maturity of the tea leaves with different $\mathrm{K}$ values. The classification accuracy of the different $K$ values is presented in Table 4, which shows that the KNN algorithm performed well at $\mathrm{K}=5$ with an accuracy of $95.53 \%$. Initially, the accuracy of the KNN was stable for $K=2$ to 4 . But after that, the testing accuracy of the KNN changed with the value of the $K$. The maximum testing accuracy of the $\mathrm{KNN}$ is at $\mathrm{K}=5$. After $K=5$, the testing accuracy decreased.

The DNN algorithm has already been used as a primary model for tea leaf maturity status classification. Apart from the DNN, the SVM and KNN models are also used to classify the maturity of the tea leaves. From Table 2, it is shown that the accuracy of the DNN model is $97 \%$, whereas the accuracy of the SVM-linear, SVM-RBF, and $\mathrm{KNN}$ are $42 \%, 94.42 \%$, and $95.53 \%$, respectively. The comparisons of models and their precision, recall, f1 score, and confusion matrix are presented in Table 5.

From Table 5, the precision of the SVM-RBF is more in each class than the SVM-linear. With a precision value of 1 for classes 1 and 2, the DNN reports a 0.96 precision value than SVM and KNN. The DNN reports 1 recall value for the class mature and immature tea class. The $f 1$ score of the DNN is higher than the $f 1$ score of the SVM and KNN. The KNN reports the second-highest precision than the SVM. The SVM linear is not performing well due to the scattered nature of the data features. The confusion matrix of the SVM-RBF denotes that the complete 22 testing mature tea leaf images are perfectly classified as mature leaf whereas the 2 immature tea leaf is classified as mature tea leaf

Table 4. Results of the KNN for different $\mathrm{K}$ values

\begin{tabular}{lll}
\hline KNN & Training KNN & Testing KNN \\
\hline KNN at $K=1$ & 1 & 1 \\
KNN at $K=2$ & 99.65 & 94.44 \\
KNN at $K=3$ & 99.65 & 94.44 \\
KNN at $K=4$ & 99.65 & 94.44 \\
KNN at $K=5$ & 99.65 & 95.53 \\
KNN at $K=6$ & 95.48 & 83.33 \\
KNN at $K=7$ & 91.31 & 72.22 \\
KNN at $K=8$ & 80.2 & 59.72 \\
KNN at $K=9$ & 82.29 & 63.83 \\
\hline
\end{tabular}


Table 5. Comparative analysis of the DNN, SVM, and KNN algorithms

\begin{tabular}{|c|c|c|c|c|c|c|c|c|c|c|}
\hline Model & Class & Precision & Recall & F1 Score & Support & $\begin{array}{l}\text { Accuracy } \\
\text { (Round Off) }\end{array}$ & Class & \multicolumn{3}{|c|}{ Confusion Matrix } \\
\hline & 0 & 0.85 & 1 & 0.92 & 22 & & 0 & 22 & 0 & 0 \\
\hline \multirow[t]{3}{*}{ SVM-RBF } & 1 & 1 & 0.92 & 0.96 & 24 & $94 \%$ & 1 & 2 & 22 & 0 \\
\hline & 2 & 1 & 0.92 & 0.96 & 26 & & 2 & 2 & 0 & 24 \\
\hline & 0 & 0.36 & 1 & 0.53 & 22 & & 0 & 22 & 0 & 0 \\
\hline \multirow[t]{3}{*}{ SVM-Linear } & 1 & 0.67 & 0.25 & 0.36 & 24 & $42 \%$ & 1 & 18 & 6 & 0 \\
\hline & 2 & 1 & 0.08 & 0.14 & 26 & & 2 & 21 & 3 & 2 \\
\hline & 0 & 0.88 & 1 & 0.94 & 22 & & 0 & 22 & 0 & 0 \\
\hline \multirow[t]{3}{*}{$\mathrm{KNN}$ at $\mathrm{K}=5$} & 1 & 1 & 0.88 & 0.93 & 24 & $96 \%$ & 1 & 3 & 21 & 0 \\
\hline & 2 & 1 & 1 & 1 & 26 & & 2 & 0 & 0 & 26 \\
\hline & 0 & 0.96 & 1 & 0.98 & 26 & & 0 & 26 & 0 & 0 \\
\hline \multirow[t]{2}{*}{ DNN } & 1 & 1 & 1 & 1 & 25 & $97 \%$ & 1 & 0 & 25 & 0 \\
\hline & 2 & 1 & 0.95 & 0.98 & 21 & & 2 & 1 & 0 & 20 \\
\hline
\end{tabular}

out of 24 immature tea leaf images. 2 tender leaves also show misclassification and reports as mature in case of SVM-RBF. A total of 4 tea leaf shows the misclassification in SVM-RBF. The total misclassification of tea leaf images in SVM-linear is 11, where 6 are immature and 5 are in the tender. In KNN, only 3 images are in the misclassification category and that is immature. With only 1 image as misclassification, the DNN model classifies 26 images as mature, 25 images as immature, and 20 images as tender. So, the Confusion matrix also reports that the DNN model outperforms. Wicaksono et al., (2019) reported 80\% accuracy for the teal leaves maturity status classification by extracting the $\mathrm{YCbCr}$ colour values from the leave images. In the present study, the DNN reported an accuracy of $97 \%$, which is more than the accuracy of the study reported by Wicaksono et al., (2019) while this accuracy is similar to the accuracy $(97.1 \%)$ reported by Sudana et al., (2020) using CNN in the mangosteen fruit.

\section{Conclusion}

The present study showed the change of tea leaf chlorophyll with the change of leafage. The DNN algorithm with an accuracy of $97 \%$ is reported as the best model for tea leaf maturity classification. This study provides a new direction to tea farming by categorizing the tea leaf images. The system will help the small-scale tea farmer to find the best tea leaf for better productivity. The system presents a new low-cost image acquisition method for tea leaf maturity classification. The overall system is authenticated and acceptable than the visual system classification. The system can be loaded into a smartphone application and can be used by any tea farmer to select the best tea for better tea productivity. The system can also be used in tea farming robots.

\section{ACKNOWLEDGEMENTS}

This research project is supported by the All India Council for Technical Education (AICTE) under RPS (NER), vide Ref. No.: File No. 8-131/RIFD/RPS-NER/ POLICY-1/2018-19 dated March 14, 2019

\section{Conflict of interest}

The authors declare that they have no conflict of interest.

\section{REFERENCES}

1. Anwar, K., Rahmanto, B., Triyasmono, L., Rizki, M. I., Halwany, W. \& Lestari, F. (2017). The influence of leaf age on total phenolic, flavonoids, and free radical scavenging capacity of Aquilaria beccariana. Research Journal of Pharmaceutical Biological and Chemical Sciences, 8, 129-133.

2. Barman, U. (2021). Deep Convolutional neural network (CNN) in tea leaf chlorophyll estimation: A new direction of modern tea farming in Assam, India. Journal of Applied and Natural Science, 13(3), 1059-1064.

3. Barman, U. \& Choudhury, R. D. (2019). Soil texture classification using multi class support vector machine. Information Processing in Agriculture.

4. Barman, U. \& Choudhury, R. D. (2020). Smartphone Image Based Digital Chlorophyll Meter to Estimate the Value of Citrus Leaves Chlorophyll using Linear Regression, LMBP-ANN and SCGBP-ANN. Journal of King Saud University-Computer and Information Sciences.

5. Barman, U. Choudhury, R. D., Sahu, D., \& Barman, G. G. (2020). Comparison of convolution neural networks for smartphone image based real time classification of citrus leaf disease. Computers and Electronics in Agriculture, 177, 105661.

6. Barman, U. Choudhury, R. D. \& Uddin, I. (n.d.). Predication of Soil pH using $\mathrm{K}$ mean Segmentation and HSV Color Image Processing.

7. Barman, U. Sarmah, A., Sahu, D. \& Barman, G. G. (2021). Estimation of tea leaf chlorophyll using MLR, ANN, SVR, and KNN in natural light condition. Proceedings of 
the International Conference on Computing and Communication Systems: I3CS 2020, NEHU, Shillong, India, 170, 287.

8. Baruah, P. (2015). Types of tea, value addition and product diversification of Indian tea. Proceedings of the First International Conference on Tea Science and Development, pp 153-159

9. Behera, S. K., Rath, A. K. \& Sethy, P. K. (2020). Maturity status classification of papaya fruits based on machine learning and transfer learning approach. Information Processing in Agriculture, 8(1), DOI:10.1016/j.inpa.2020.0 5.0 03

10. Mubin, N. A., Nadarajoo, E., Shafri, H. Z. M. \& Hamedianfar, A. (2019). Young and mature oil palm tree detection and counting using convolutional neural network deep learning method. International Journal of Remote Sensing, 40(19), 7500-7515.

11. Najeeb, T. \& Safar, M. (2018). Dates maturity status and classification using image processing. 2018 International Conference on Computing Sciences and Engineering
(ICCSE), 1-6.

12. Pourdarbani, R., Sabzi, S., Kalantari, D., Karimzadeh, R., Ilbeygi, E. \& Arribas, J. I. (2020). Automatic nondestructive video estimation of maturation levels in Fuji apple (Malus Malus pumila) fruit in orchard based on colour (Vis) and spectral (NIR) data. Biosystems Engineering, 195, 136-151.

13. Sood, M., \& Singh, P. K. (2020). Hybrid system for detection and classification of plant disease using qualitative texture features aAnalysis. Procedia Computer Science, 167, 1056-1065.

14. Sudana, O., Bayupati, I. P. A., \& Yudiana, D. G. (2020). Classification of maturity level of the mangosteen using the Convolutional neural network (cnn) method. Learning, 135.

15. Wicaksono, B. A., Novamizanti, L., \& Ibrahim, N. (2019). Tea leaf maturity levels based on ycbcr color space and clustering centroid. Journal of Physics: Conference Series, 1367(1), 012028. 